\title{
Arsenic Trioxide in the Treatment of Relapsed and Refractory Acute Promyelocytic Leukemia
}

Key words: acute promyelocytic leukemia, all-trans retinoic acid, arsenic trioxide, PML-RAR $\alpha$

Acute promyelocytic leukemia (APL) is a distinct subtype of acute myeloblastic leukemia affecting approximately $10 \%$ of adult patients (1). Patients with APL show an extreme bleeding diathesis caused by disseminated intravascular coagulation and excessive fibrinolysis. APL cells in the vast majority of patients have a chromosomal translocation $\mathrm{t}(15 ; 17)$ that produces the fusion gene consisting of promyelocytic leukemia $(P M L)$ and retinoic acid receptor $\alpha(R A R \alpha)$. Introduction of all-trans retinoic acid (ATRA) has significantly improved the clinical outcome in APL patients (1). In the history of cancer therapy, ATRA is the first successive molecular targeted agent against PML-RAR $\alpha$. More than $90 \%$ of APL patients treated with ATRA and cytotoxic chemotherapy achieve a complete remission (CR) and approximately $70 \%$ of patients have a relapse-free survival (2). However, about $15-25 \%$ of the patients eventually relapse and are refractory to further ATRA and chemotherapy.

In China, the initial studies of arsenic trioxide in patients with newly diagnosed and relapsed APL documented a marked clinical efficacy $(3,4)$. The multiple mechanisms behind the effects of arsenic in APL include induction of apoptosis, stimulation of differentiation, and inhibition of angiogenesis (1). Since arsenic functions through mechanisms different from ATRA, arsenic can induce a CR rate of more than $80 \%$ in relapsed patients after treatment with ATRA (Table 1). Furthermore, pooled results in the USA trials showed molecular remission, as measured by PML-RAR $\alpha$ transcript, in $83 \%$ of 40 relapsed patients $(5,6)$. In Japan, the investigators in Hamamatsu University School of Medicine used arsenic for relapsed APL patients and showed a high CR rate (7). The Ministry of Health and Welfare of Japan approved arsenic trioxide $\left(\right.$ Trisenox ${ }^{\oplus}$ ) for the treatment of relapsed and refractory APL in October 2004.

The adverse effects associated with arsenic include leukocytosis, APL differentiation syndrome, QTc prolongation on electrocardiogram, peripheral neuropathy, rash, and gastrointestinal symptoms. These are self-limiting in most patients, although cases of sudden death, probably of cardiac origin, and severe hepatotoxicity have been reported (3-7). In this issue, Shinjo et al (8) reported delayed recovery of normal hematopoiesis in the arsenic treatment of relapsed and refractory APL patients.

\section{See also p 818.}

Durations of neutropenia and thrombocytopenia during arsenic treatment were significantly longer than those in ATRA therapy. They compared blood cells recovery in relapsed and refractory patients treated with arsenic and newly diagnosed patients treated with ATRA. Nonetheless, it is important to predict blood cells recovery in the prophylaxis for infection and bleeding. Although no severe marrow hypoplasia was found in other studies (3-6), hematological toxicities observed in Japanese patients might be associated with older ages and longer durations of arsenic treatment than those in other studies (Table 1). We must carefully use arsenic for relapsed APL patients in regard to blood cell recovery.

Currently, arsenic is considered as first-line therapy for

Table 1. Arsenic Trioxide for Relapsed or Refractory APL Patients after Treatment with ATRA

\begin{tabular}{|c|c|c|c|c|c|c|}
\hline Author & Year & No. of patients & Age & CR $(\%)$ & Treatment* & Days to $\mathrm{CR}$ \\
\hline Shen ZX (3) & 1997 & 15 & $40(14-53)$ & $14(93)$ & $34(28-54)$ & $33(28-54)$ \\
\hline Soignet SL (5) & 1998 & 12 & $34(9-75)$ & $11(92)$ & $33(12-39)$ & $47(24-83)$ \\
\hline Niu C (4) & 1999 & 47 & $38(7-55)$ & $40(85)$ & - & $31(17-76)$ \\
\hline Soignet SL (6) & 2001 & 40 & - & $34(85)$ & $35(20-85)$ & $59(28-85)$ \\
\hline Ohnishi K (7) & 2002 & 14 & $51(23-65)$ & $11(78)$ & $43(27-60)$ & $43(27-60)$ \\
\hline
\end{tabular}

*: The median duration of therapy in patients who responded to treatment. 
relapsed APL patients (1). Since arsenic does not appear to completely eradicate leukemic cells, the best postremission therapy remains to be examined. The relatively good outcome supports autologous stem cell transplantation in patients in second CR after arsenic therapy. However, the study in Hamamatsu University may be a warning of problems with stem cell harvest after arsenic treatment. While other neoplasms such as multiple myeloma, myelodysplastic syndrome and adult T-cell leukemia possibly benefit from arsenic, myelosuppression also appears to be the predominant toxicity in patients with these diseases and solid tumors.

Norio AsOU

Department of Hematology, Kumamoto University School of Medicine,

1-1-1 Honjo, Kumamoto 860-8556

\section{References}

1) Ohno R, Asou N, Ohnishi K. Treatment of acute promyelocytic leukemia: strategy toward further increase of cure rate. Leukemia 17: 14541463,2003

2) Asou N, Adachi K, Tamura J, et al. Analysis of prognostic factors in newly diagnosed acute promyelocytic leukemia treated with all-trans retinoic acid and chemotherapy. Japan Adult Leukemia Study Group. J Clin Oncol 16: 78-85, 1998.

3) Shen ZX, Chen GQ, Ni JH, et al. Use of arsenic trioxide (As2O3) in the treatment of acute promyelocytic leukemia (APL): II. Clinical efficacy and pharmacokinetics in relapsed patients. Blood 89: 3354-3360, 1997.

4) Niu C, Yan H, Yu T, et al. Studies on treatment of acute promyelocytic leukemia with arsenic trioxide: remission induction, follow-up, and molecular monitoring in 11 newly diagnosed and 47 relapsed acute promyelocytic leukemia patients. Blood 94: 3315-3324, 1999.

5) Soignet SL, Maslak P, Wang ZG, et al. Complete remission after treatment of acute promyelocytic leukemia with arsenic trioxide. N Engl J Med 339: 1341-1348, 1998.

6) Soignet SL, Frankel SR, Douer D, et al. United States multicenter study of arsenic trioxide in relapsed acute promyelocytic leukemia. J Clin Oncol 19: 3852-3860, 2001.

7) Ohnishi K, Yoshida H, Shigeno K, et al. Arsenic trioxide therapy for relapsed or refractory Japanese patients with acute promyelocytic leukemia: need for careful electrocardiogram monitoring. Leukemia 16: 617-622, 2002.

8) Shinjo K, Takeshita A, Sahara N, et al. Delayed recovery of normal hematopoiesis in arsenic trioxide treatment of acute promyelocytic leukemia: a comparison to all-trans retinoic acid treatment. Intern Med 44: 818-824, 2005. 